\title{
Ellagic acid inhibits human glioblastoma growth in vitro and in vivo
}

\author{
DONGLIANG WANG, QIANXUE CHEN, YINQIU TAN, BAOHUI LIU and CHAO LIU \\ Department of Neurosurgery, Renmin Hospital of Wuhan University, Wuhan, Hubei 430060, P.R. China
}

Received June 29, 2016; Accepted August 23, 2016

DOI: $10.3892 /$ or.2016.5331

\begin{abstract}
Ellagic acid (EA) is present in various fruits and plants and has recently been found to possess anticarcinogenic effects. The objective of this study was to investigate the anti-glioblastoma effect of EA and its mechanisms in vitro and in vivo. We first studied the anticancer activity of EA in U87 and U118 human glioblastoma cell lines. The cell viability and cell proliferation were examined by Cell Counting Kit-8 (CCK-8) assay and 5-ethynyl-2'-deoxyuridine staining respectively. The cell cycle was detected with propidium iodide staining method by flow cytometry and the DNA damage of the cells caused by EA exposure was evaluated by detection of $\gamma-\mathrm{H} 2 \mathrm{AX}$ foci. Then we examined the effect of EA on tumor growth in glioblastoma xenografted mice, and expression of Akt and Notch signaling and their target gene products were detected by immunohistochemistry and western blot analysis. As a result, we found that the cell viability and proliferation of glioblastoma cells treated with EA were significantly suppressed compared with the control; EA significantly increased the proportion of cells in the $S$ phase accompanied by a decrease in the population in the $\mathrm{G} 1$ and $\mathrm{G} 2 / \mathrm{M}$ phase in both cell lines. Meanwhile, the level of DNA damage in the EA-treated group was significantly higher than that of the control. Treatment of glioblastoma in xenografted mice by EA led to a significant suppression in tumor growth. EA upregulated the expression
\end{abstract}

Correspondence to: Professor Qianxue Chen, Department of Neurosurgery, Renmin Hospital of Wuhan University, 238 Jiefang Road, Wuhan, Hubei 430060, P.R. China

E-mail: qxchen430@sina.com

Abbreviations: EA, ellagic acid; Bcl-2, B-cell lymphoma 2; CDK, cyclin-dependent kinase; $\gamma$-H2AX, phosphorylated histone H2AX; PCNA, proliferating cell nuclear antigen; MMPs, matrix metalloproteinases; PI, propidium iodide; DMEM, Dulbecco's modified Eagle's medium; PBS, phosphate-buffered saline; FBS, fetal bovine serum; EdU, 5-ethynyl-2'-deoxyuridine; IHC, immunohistochemistry; WB, western blot analysis; EMT, epithelial-mesenchymal transition; ECM, extracellular matrix; DSBs, double-strand breaks

Key words: glioblastoma, ellagic acid, proliferation, metastasis, Akt, Notch of E-cadherin and inhibited the expression of Snail, matrix metalloproteinase (MMP)-2 and MMP-9. EA also inhibited the expression of Bcl-2, cyclin D1, cyclin-dependent kinase (CDK)2 and CDK6 in U87 xenograft tissues. In addition, significant suppression of Akt and Notch was found in the xenografts of the tumor-bearing mice treated with EA. These data indicate that EA can suppress glioblastoma proliferation and invasion by inhibiting the Akt and Notch signaling pathways, which suggests that EA may be beneficial for the treatment of glioblastoma.

\section{Introduction}

Glioma is a common type of primary tumor in the central nervous system. It includes predominantly astrocytic, oligodendroglial, ependymal, neuronal and mixed neuronal-glial tumors. As a grade IV glioma classified by the World Health Organization, glioblastoma multiforme is considered the most malignant and invasive subtype with a median survival time of 14.6 months even after a three-pronged approach comprised of surgical resection, radiation and chemotherapy (1). In fact, approximately $95 \%$ of patients suffering from glioblastoma succumb to this disease within 5 years after initial diagnosis (2). Therefore, seeking novel strategies to treat glioblastoma appears to be crucial.

In recent years, the use of phytochemicals as antitumor agents has received increasing attention. A number of plant-derived compounds such as puerarin, gingerol and silibinin have been reported to have anticarcinogenic effects (3-5). Ellagic acid (EA), as a type of natural dietary polyphenol compound, exists in several fruits and plants such as walnuts, blackberries, Pericarpium granati, cranberries, raspberries, strawberries and grapes (6). As a dimeric derivative of gallic acid, EA (its chemical structural formula is shown in Fig. 1A) has been found to have a variety of pharmacological properties, such as antioxidant, antifibrosis, chemoprevention and anticarcinogenic (7-9). The crude extract of EA from Pericarpium granati has been widely applied in cooking and clinically in traditional Chinese medicine for over two thousand years. The anticarcinogenic effect of EA has also been revealed in several types of tumors (10-15), while the effect of EA on glioblastoma has rarely been reported. Here, in the present study, we aimed to observe the anti-glioblastoma effect of EA and investigate its molecular mechanisms in both glioblastoma cell lines and glioblastoma xenografts in Balb/c nude mice. 
The PI3K/Akt signaling pathway plays an important part in cell survival and proliferation, and it is usually activated eccentrically in the later stages of human glioblastoma. The dysregulation of the PI3K/Akt signaling axis, as well as PTEN, in both glioblastoma initiation and progression, has illustrated the signaling mechanisms which cause the development of glioblastoma. What is more, Guo et al revealed that resveratrol could suppress glioblastoma carcinogenesis in vitro and in vivo (16).

As a highly conserved pathway throughout evolution, the Notch signaling pathway plays a major role in tumor cell survival, differentiation, and proliferation (17). In malignant tumors, the Notch signaling pathway has been demonstrated to function with both oncogenic and anticarcinogenic effects according to different cell types (18). An increasing amount of evidence has shown the importance of the modified Notch signaling pathway in the growth, apoptosis and differentiation in several malignant tumors including glioblastoma. The Notch1 signaling pathway plays critical roles in glioma formation by facilitating exocrine lineage development and neural progenitor cell self-renewal in in vivo experiments. Moreover, it has been confirmed that the Notch signaling pathway is upregulated in glioblastoma.

The cell cycle and apoptosis play an important part in tumorigenesis. B-cell lymphoma 2 (Bcl-2) family members play a dominant role in the process of cell apoptosis. During tumor growth, cell cycle progression is enhanced. Signaling pathways, to a certain extent, regulate cells to proliferate or remain quiescent, which may link cell environment information to specific stages of the cell cycle. The combination of cyclin-dependent kinase (CDK) catalytic subunits and cyclin partners or CDK inhibitors could regulate the activity of CDK. As a result, cell proliferation is influenced by antiproliferative and mitogenic signals through ubiquitin-dependent degeneration of cyclins or CDK inhibitors and transcriptional regulation. Thus, identifying molecular targets to promote suppression of the the cell cycle in cancer cells may be an effective anticarcinogenic method.

\section{Materials and methods}

Reagents. Dulbecco's modified Eagle's medium (DMEM) and phosphate-buffered saline (PBS) were purchased from Genom Co., Ltd. (Hangzhou, China). Fetal bovine serum (FBS) was obtained from Tianhang Biotechnology Co., Ltd. (Deqing, China). EA was purchased from Cayman Chemical Co. (Ann Arbor, MI, USA). DMSO was acquired from MP Biomedicals (Solon, OH, USA). The Cell Counting Kit-8 (CCK-8) was obtained from Dojindo China Co., Ltd. (Beijing, China). The Cell-Light EdU DNA cell proliferation kit was purchased from RiboBio Co., Ltd. (Guangzhou, China). A bicinchoninic acid (BCA) kit for protein determination, a cell cycle and apoptosis analysis kit and Hoechst 33342 solution were obtained from Beyotime Institute of Biotechnology (Jiangsu, China). Antibodies against Bcl-2, cyclin D1, phosphorylated histone $\mathrm{H} 2 \mathrm{AX}(\gamma-\mathrm{H} 2 \mathrm{AX})$, proliferating cell nuclear antigen (PCNA), Ki67, CDK2, CDK6, E-cadherin, Akt, Snail, Notch1, matrix metalloproteinase (MMP)-2, $\beta$-actin and MMP-9 were obtained from Cell Signaling Technology, Inc. (Danvers, MA, USA). Alex Fluor 680/790 labeled goat anti-rabbit/mouse IgG was purchased from LI-COR Biosciences (Lincoln, NE, USA). Unless otherwise stated, all other materials were purchased from AntGene Biotechnology Co., Ltd. (Wuhan, China).

Cell lines and animals. The human glioblastoma U87 and U118 cell lines were obtained from the Shanghai Cell Bank, Chinese Academy of Sciences (Shanghai, China). Balb/c nude mice aged 5-6 weeks were purchased from the Hunan SJA Laboratory Animal Co., Ltd. (Changsha, China). All experimental procedures involving animals in our study were performed in accordance with the guidelines of the Animal Experiment Center of Wuhan University and approved by the Animal Care and Use Ethics Committee of Wuhan University. All experimental animals were housed in cages and maintained at a humidity of $50 \pm 5 \%$ and a temperature of $27 \pm 1^{\circ} \mathrm{C}$ in a controlled animal facility under diurnal lighting conditions (12-h light/dark cycle) with free access to food and water.

Cell culture and xenografted models. The U87 and U118 cell lines were grown and maintained in DMEM supplemented with $10 \%$ FBS and $1 \%$ antibiotic mixture (penicillin and streptomycin) in a constant temperature incubator set at $37^{\circ} \mathrm{C}$ abounding with $5 \% \mathrm{CO}_{2}$. The culture medium was changed every 3 days. The cells were trypsinized when they reached $\sim 90 \%$ confluency. In order to examine the anticarcinogenic potential of EA, a U87 MG xenograft model was constructed. The cells were seeded in a cell culture dish and were trypsinized when the cells reached a logarithmic growth period. Then a cell suspension with serum-free medium at a density of $2 \times 10^{7}$ cells $/ \mathrm{ml}$ was prepared. Subsequently, $2 \times 10^{6}$ cells were injected subcutaneously into the flanks of Balb/c nude mice as approved in the protocol. The mice were randomly divided into two groups, each group containing 6 mice. The experimental group was treated with EA $(40 \mu \mathrm{g} / \mathrm{g}$ body weight) through gavage, from Monday to Friday for 4 weeks, once daily. The control group was treated with normal saline by gavage in the same way. When the experiment finished, all mice were euthanized and tumor xenografts were dissected for final volume and wet weight and immunohistochemistry (IHC) and western blot analysis (WB) were performed.

\section{Antitumor activity in vitro}

CCK- 8 test. The effect of EA on the cell viability of human glioblastoma U87 and U118 cells was assessed by CCK-8 assay. According to instructions, the cells were seeded in a 96-well plate at a density of $0.8 \times 10^{4}$ cells/well, incubated for $12 \mathrm{~h}$ and treated with various concentrations of EA $(0-200 \mu \mathrm{M})$ for 24 , 48 and 72 h, separately. At designated time-points (24, 48 and $72 \mathrm{~h}$ ), $100 \mu \mathrm{l}$ of EA-free medium containing $5 \mu \mathrm{l} \mathrm{CCK}-8$ solution was added into each well and incubation followed for $1 \mathrm{~h}$. Then the absorbance of the cells at $450 \mathrm{~nm}$ was detected on a Multiskan spectrum microplate spectrophotometer. The experiment was repeated for three wells in each group.

$E d U$ staining. The procedure was performed following the product specifications. U87 and U118 cells were seeded in a 96-well plate and subsequently treated with or without EA for $48 \mathrm{~h}$. All cells were exposed to $10 \mu \mathrm{M}$ 5-ethynyl-2'deoxyuridine (EdU) for $12 \mathrm{~h}$ in an incubator at $37^{\circ} \mathrm{C}$ and then washed for 5 min twice with PBS. After being fixed with $4 \%$ 
paraformaldehyde for $30 \mathrm{~min}$ at room temperature, the cells were treated with $0.5 \%$ Triton $\mathrm{X}-100$ for $10 \mathrm{~min}$ and rinsed with PBS for $5 \mathrm{~min}$. Thenceforth, the cells were treated with $100 \mu \mathrm{l}$ of $1 \mathrm{X}$ Apollo ${ }^{\circledR}$ Reaction Cocktail for $30 \mathrm{~min}$ in the dark at room temperature. Then the cells were treated with $0.5 \%$ Triton X-100 for $20 \mathrm{~min}$ and rinsed with PBS for $5 \mathrm{~min}$. Subsequently, the cells were incubated for 30 min with $1 \mathrm{X}$ Hoechst 33342 in order to dye the cell nuclei. The images of EdU and Hoechst fluorescence in the cells were captured using an Olympus fluorescence microscope (Olympus, Tokyo, Japan). Five random fields of each well were observed. The number of EdU and Hoechst-positive cells were counted using ImageJ software and the EdU labeling index was calculated.

Cell cycle distribution is assessed by flow cytometry. The effect of EA on the glioblastoma cell cycle distribution was assessed using a cell cycle and apoptosis analysis kit following the manufacturer's instructions. In brief, the U87 and U118 cells were treated with or without EA for $48 \mathrm{~h}$. Then $\sim 1 \times 10^{6}$ cells were trypsinized, collected by centrifugation at $1,000 \mathrm{rpm}$ for 5 min and washed using ice cold PBS. After being fixed with ice cold $70 \%$ ethanol for $12 \mathrm{~h}$ at $4^{\circ} \mathrm{C}$, the cells were collected by centrifugation at $100 \mathrm{rpm}$ for $5 \mathrm{~min}$ and then washed with ice cold PBS. Subsequently, $0.5 \mathrm{ml}$ of propidium iodide (PI) staining solution was added and incubation followed at $37^{\circ} \mathrm{C}$ in the dark for $30 \mathrm{~min}$. The cell cycle distribution was analyzed on a flow cytometer (BD Biosciences, San Diego, CA, USA).

Histone $H 2 A X$ antibody detects nuclear $H 2 A X$ protein by immunofluorescent analysis. The U87 and U118 cell lines were trypsinized and seeded in 6-well culture plates on coverslips and incubated for $12 \mathrm{~h}$ and then treated with EA or without for $48 \mathrm{~h}$. The cells growing on the coverslips were fixed in $4 \%$ formaldehyde in PBS for $30 \mathrm{~min}$ at room temperature. For detection of $\gamma-\mathrm{H} 2 \mathrm{AX}$ foci, the coverslips were incubated with anti- $\gamma-\mathrm{H} 2 \mathrm{AX}$ rabbit monoclonal antibody overnight at $4^{\circ} \mathrm{C}$. After being washed twice with PBS, the cells were incubated with fluorescein isothiocyanatelabeled goat anti-rabbit secondary antibody for $1 \mathrm{~h}$, then washed twice with PBS. The nuclei were counterstained with Hoechst 33342 in PBS for 30 min before coverslips were covered by anti-fade solution and finally observed under the Olympus fluorescence microscope. It was surmised that the positive foci were those that were clear with easily diacritical dots of certain brightness.

Matrigel invasion assay. Transwell filters with $8-\mu$ m pores (BD Biosciences) were placed into 24-well culture plates. The bottom surface of the filters was coated with $50 \mu \mathrm{l}$ of Matrigel diluted in PBS and dried at $37^{\circ} \mathrm{C}$ for $1 \mathrm{~h}$. The bottom surface of the filters was then washed with migration assay buffer (DMEM supplemented with $0.1 \%$ fatty-acid free bovine serum albumin). Approximately $500 \mu \mathrm{l}$ of migration assay buffer was placed at the bottom of each filter, and 60,000 cells suspended in migration assay buffer were placed on top of the filters. Cells were allowed to adhere for $0.5 \mathrm{~h}$ before drug treatment. Cells were then allowed to migrate for an additional $23.5 \mathrm{~h}$ in the cell incubator. To stop migration, filters were fixed and stained with crystal violet. The top of the filters was wiped clean before imaging on an Olympus fluorescence microscope.
The cell invasion was quantified by counting the number of cells per field in five random fields.

\section{Antitumor activity in vivo}

IHC. Briefly, at the end of the animal experiment, xenograft tissues were collected and fixed with $10 \%$ formalin, then embedded in paraffin and sectioned. Antigens were retrieved using a routine antigen retrieval method and washed for $5 \mathrm{~min}$ twice with PBS. Ultra V Block was added to the tissues, incubated for $5 \mathrm{~min}$ at room temperature and washed twice again with PBS. Then, the sections were incubated with different primary antibodies (CDK6, PCNA, CDK2, Ki-67, MMP-9, MMP-2, Bcl-2, cyclin D1, Snail, Akt, Notch1 and E-cadherin) for $2 \mathrm{~h}$ at $37^{\circ} \mathrm{C}$ in a humidified chamber. After being washed with PBS twice, a secondary antibody was added and the sections were incubated at room temperature for $0.5 \mathrm{~h}$. Subsequently, each section was washed for $5 \mathrm{~min}$ with PBS thrice and alkaline phosphatase working solution was added to the sections followed by incubation for $20 \mathrm{~min}$ at $37^{\circ} \mathrm{C}$ according to manufacturer's instructions. Finally, the sections were coverslipped and imaged.

$W B$. Xenograft tissue lysates were subjected to SDS-PAGE, and then gels were blotted on polyvinylidene difluoride membranes (Millipore Corp., Billerica, MA, USA). The blots were blocked using TBS blocking solution containing 5\% powdered milk at room temperature by shaking for $2 \mathrm{~h}$ and then the membranes were incubated with a primary antibody diluted according to instructions at $4^{\circ} \mathrm{C}$ overnight on a shaking table. The next day, the membranes were washed with TBST three times and incubated with a secondary antibody solution which was horseradish peroxidase-conjugated IgG $(1: 5,000)$ at room temperature in the dark for $1 \mathrm{~h}$. Subsequently, the membranes were washed with TBST three times. Finally, protein antibody complexes were measured by LI-COR Odyssey infrared imaging system (LI-COR Biosciences).

Statistical analysis. The values are represented as the mean \pm standard deviation for three independent experiments. The two-tailed Student's t-test was used to detect the significance between two experimental groups, and significance in more than two groups was determined using one-way analysis of variance. GraphPad software (ver. 5.02; GraphPad Software, Inc., La Jolla, CA, USA) was used for all statistical analyses. Statistical significant differences were established when $\mathrm{P}<0.05$.

\section{Results}

\section{Antitumor activity in vitro}

EA inhibits the cell viability of U87 and U118 cell lines. As shown in Fig. 1B and C, EA markedly decreased the cell viability in the U87 and U118 cell lines in a time- and dose-dependent manner. After incubation for $48 \mathrm{~h}$, the $\mathrm{IC}_{50}$ values (concentration of the drug resulting in a $50 \%$ reduction in cell viability) of EA against the cell viability of U87 and U118 cell lines were 91.2 and $98.6 \mu \mathrm{M}$, respectively.

EA suppresses the proliferation of glioblastoma cells. In the EdU assay, a marked suppression of cell proliferation was 
A<smiles>O=C1Oc2c(O)c(=O)oc3c(O)c(O)cc1c23</smiles>

Ellagic acid
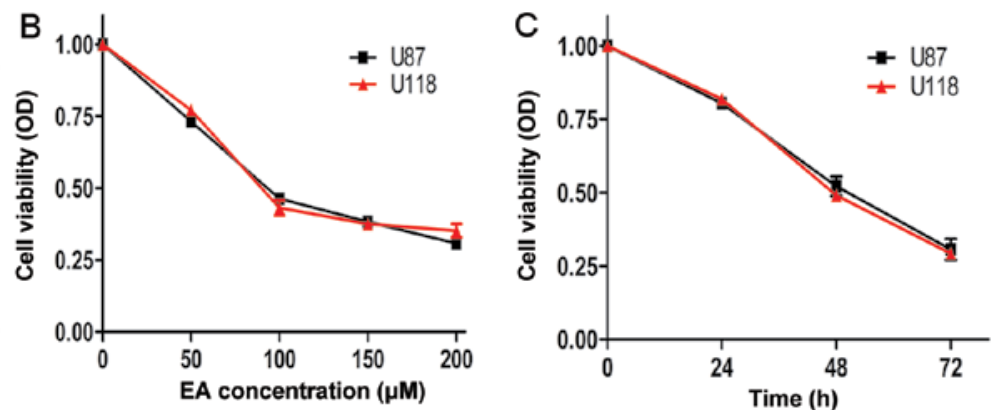

Figure 1. (A) Chemical structure of ellagic acid (EA). (B) The cell viability of U87 and U118 cell lines treated with different concentrations of EA for $48 \mathrm{~h}$ (C) The cell viability of U87 and U118 cells treated with $100 \mu \mathrm{M}$ of EA at different time-periods. Values are represented as the mean \pm standard deviation for three independent experiments.

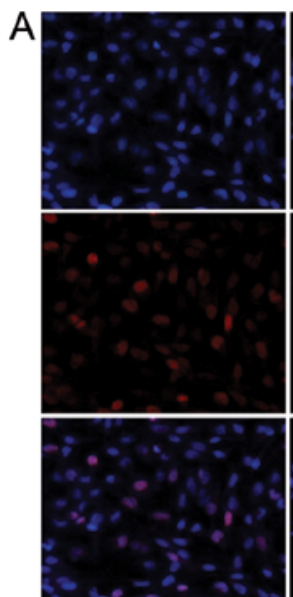

Control
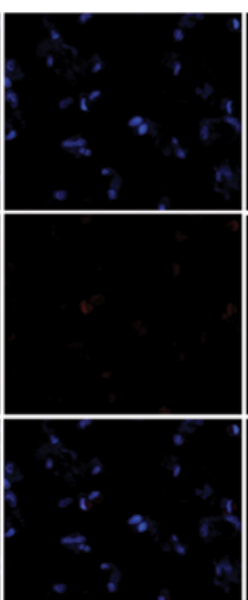

EA
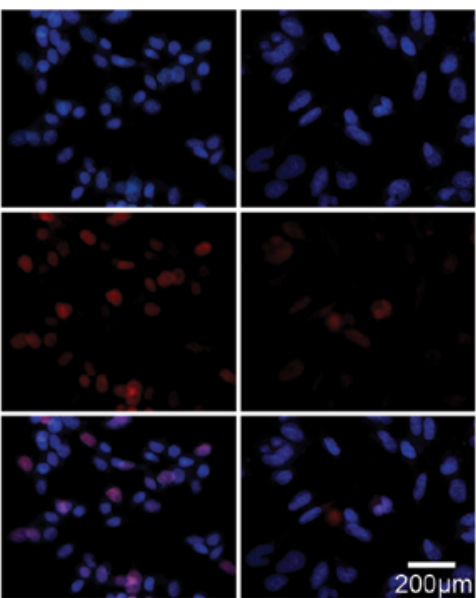

Control

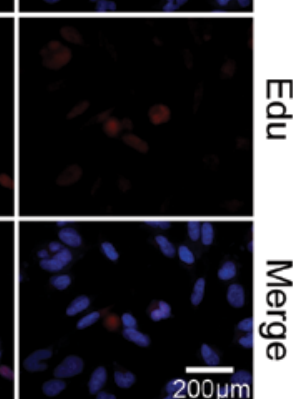

EA

U87 MG

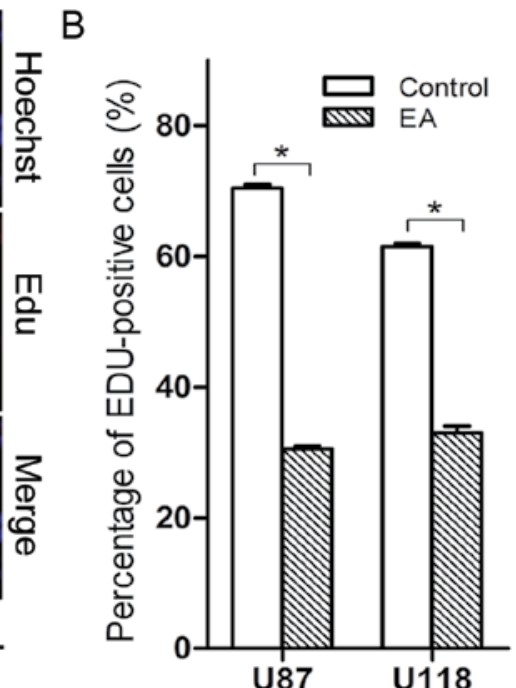

Figure 2. Ellagic acid (EA) suppresses cell proliferation in U87 and U118 cell lines. (A) Proliferating U87 and U118 cells treated with EA or without were labeled with EdU (red). The cell nuclei were dyed by Hoechst 33342 solution (blue). The images are the results observed under fluorescence microscopy (x400). (B) The percentage of EdU-positive cells treated with EA or without in U87 and U118 cells were compared. "P<0.05 represents statistical significance.
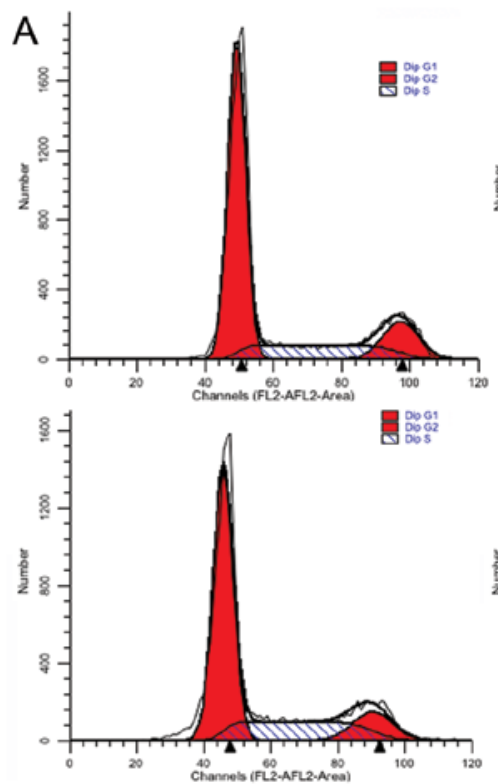

U87
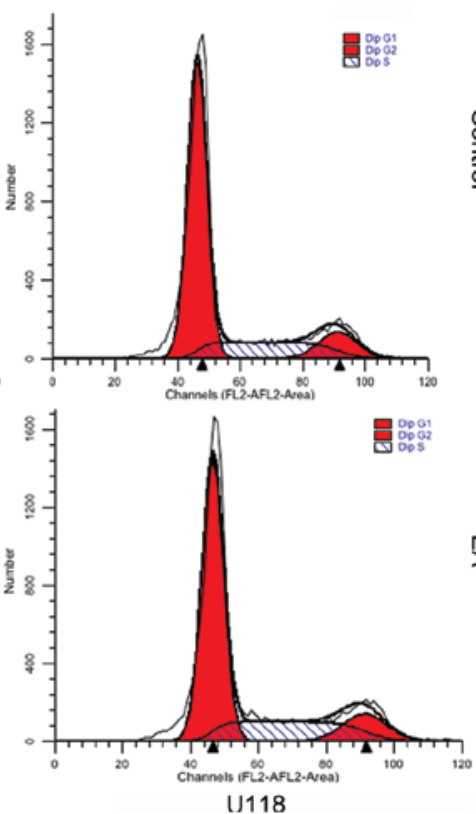

$\stackrel{\text { 윰 }}{\underline{\underline{z}}}$

m

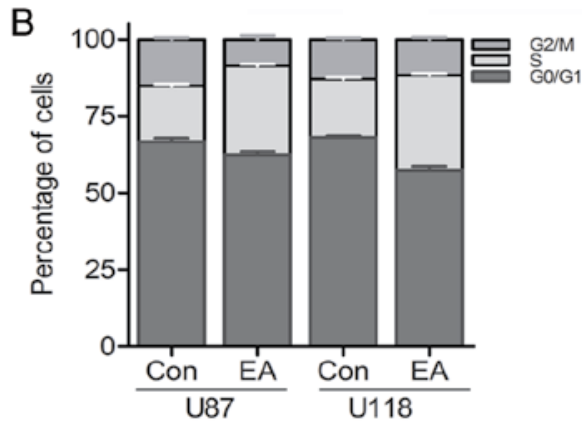

Figure 3. Ellagic acid (EA) disturbs normal cell cycle progression in U87 and U118 cell lines. (A) The DNA content of cells in different phases of the cell cycle was determined using flow cytometry in the control and experimental group. (B) The percentages of U87 and U118 cells in different phases of the cell cycle were quantified after treatment with EA or without. Results are represented as the mean \pm standard deviation. 


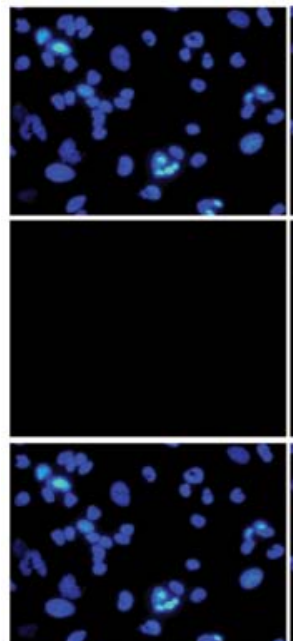

Control
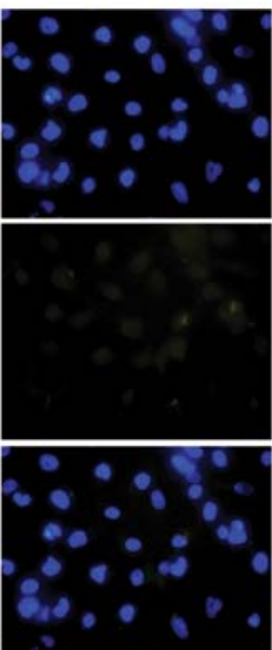

EA

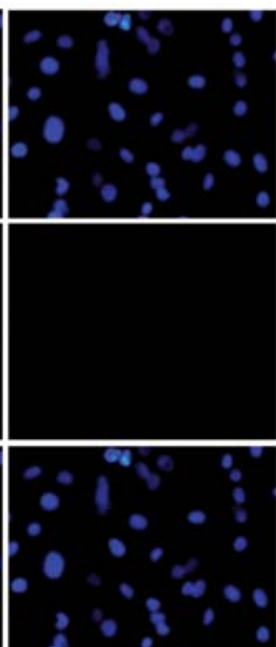

Control
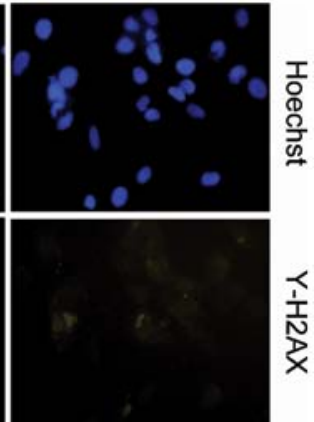

U87 MG

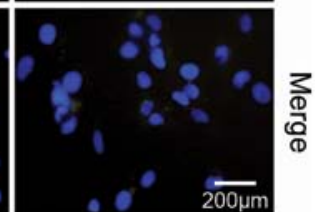

U118 MG

Figure 4. Ellagic acid (EA) induces DNA damage in glioblastoma cells. The U87 and U118 cells treated with EA or without for $48 \mathrm{~h}$ were stained with $\gamma$-H2AX antibody (green). The cell nuclei were dyed with Hoechst 33342 solution (blue) followed by immunofluorescence (x400).

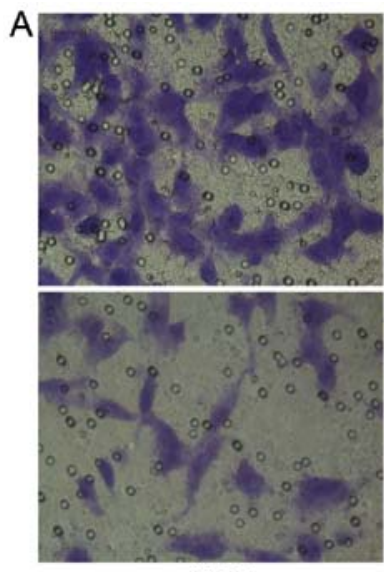

U87

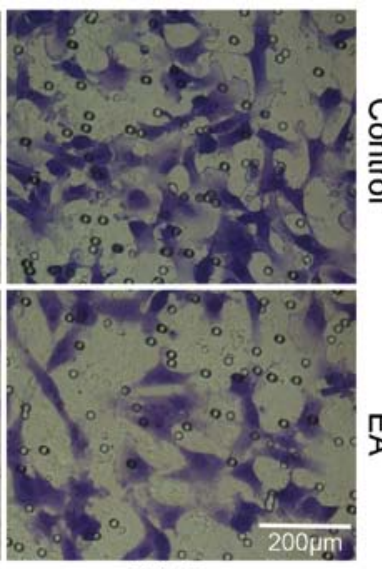

U118

\section{B}

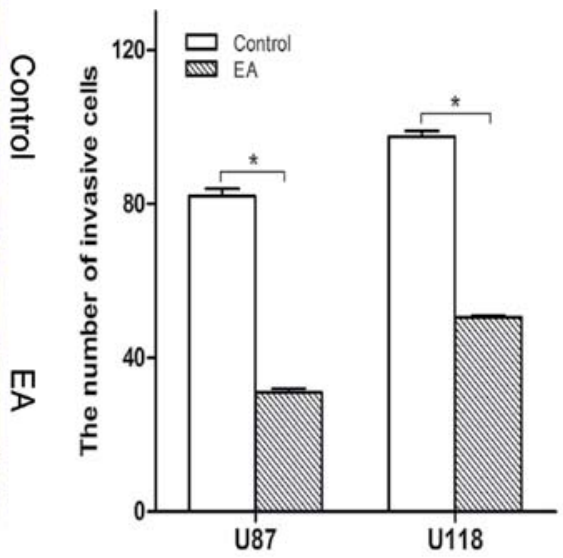

Figure 5. Ellagic acid (EA) affects glioblastoma cell invasion. (A) Transmembrane U87 and U118 cells treated with EA or without for 24 h were stained with crystal violet (x400). (B) The number of U87 and U118 cells in the control and experimental group were quantified. Results are represented as the mean \pm standard deviation. ${ }^{*} \mathrm{P}<0.05$ indicates statistical significance.

observed in both the U87 and U118 cell lines treated with $100 \mu \mathrm{M}$ of EA at $48 \mathrm{~h}$ (Fig. 2). More specifically, the number of cell nuclei with thymidine analog incorporated into newly synthesized DNA significantly decreased after treatment with EA. The percentage of stained nuclei in total cells treated with EA was lower than that noted in the control $(\mathrm{P}<0.05)$.

EA affects the cell cycle progression of glioblastoma cells. For the purpose of detecting the possible mechanisms of EA anti-proliferation, we evaluated the cell cycle distribution of both cell lines with or without EA by flow cytometry. As shown in Fig. 3, cultivating U87 and U118 cells with EA respectively for $48 \mathrm{~h}$ led to a 10.89 and $11.54 \%$ increase separately in the percentage of cells in the $S$ phase compared with the control group, while accompanied by a persistent decrease in the percentage of cells in the G1 and G2/M phases, which revealed that EA could induce cell cycle arrest at the $S$ phase in both glioblastoma cell lines.
EA leads to DNA damage in glioblastoma cells. As exhibited in Fig. 4, the double-strand breaks (DSBs) and consequent $\gamma$-H2AX foci spots induced by EA were significantly increased compared with the control group at $48 \mathrm{~h}$. The results demonstrated that EA leads to the lethality of DSBs in glioblastoma cells.

EA inhibits the invasion of glioblastoma cells. As shown in Fig. 5, EA inhibited the cell invasion in U87 and U118 cells [control $(82 \pm 3),(97 \pm 6)$ vs. EA treatment $(31 \pm 1),(50 \pm 4)$, $\mathrm{P}<0.05]$. These results demonstrated that EA decreases glioma cell invasion.

\section{Antitumor activity in vivo}

EA suppresses the growth of U87 xenografted tumors in Balb/c nude mice. The influence of EA on the growth of glioblastoma U87 xenografted tumors in Balb/c nude mice was evaluated. We observed that EA suppressed the volume and 

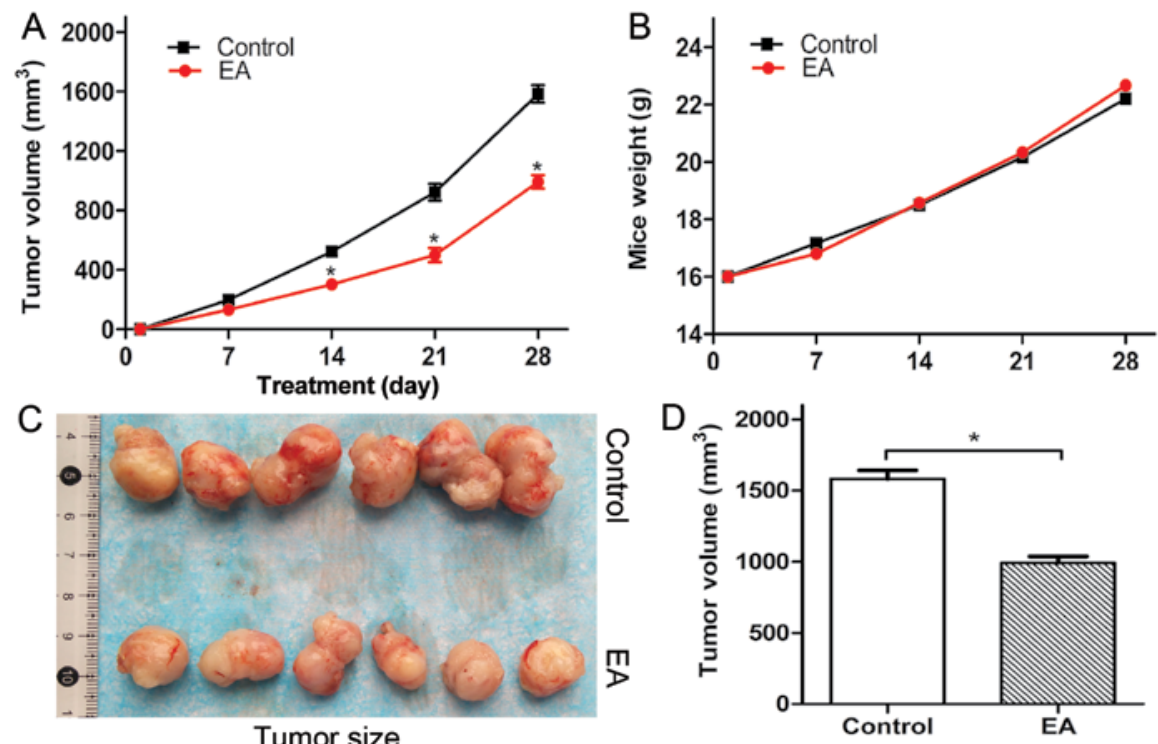

Figure 6. Ellagic acid (EA) inhibits the growth of U87 xenografted tumors in Balb/c nude mice. (A) Approximately $2 \times 10^{6}$ U87 cells were subcutaneously implanted into the flanks of Balb/c nude mice. Then the mice were randomly divided into two groups, each group containing 6 mice. Xenograft tumor volumes of the experimental and control group were measured weekly. (B) The body weight of tumor-bearing mice was recorded once per week. (C) Images of the xenografts after isolation of the control and EA-treated mice. (D) The tumor volumes of the xenografts from the experimental and control group were quantified. Results are represented as the mean \pm standard deviation. ${ }^{*} \mathrm{P}<0.05$ indicates statistical significance.

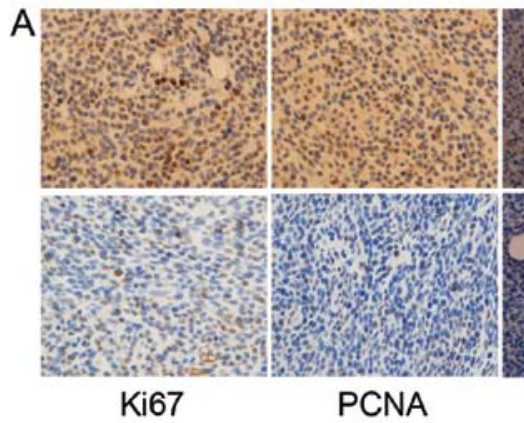

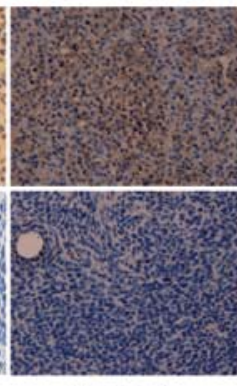

Notch1

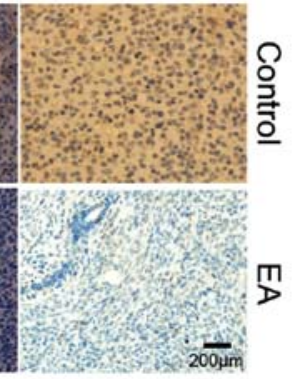

Akt
B

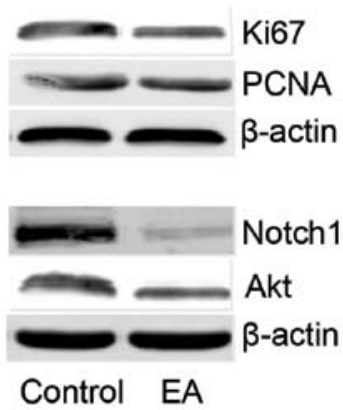

Control EA
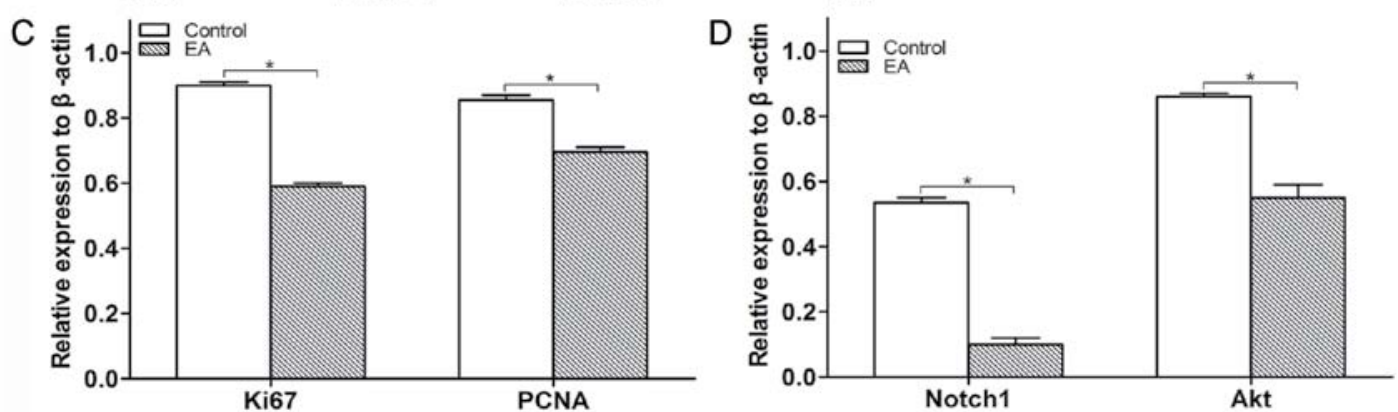

Figure 7. (A) Expression of proliferating cell nuclear antigen (PCNA), Ki67, Akt and Notch1 in xenografts derived from the experimental and control group was evaluated using immunohistochemistry. (B-D) The protein expression levels of PCNA, Ki67, Akt and Notch1 in xenografts derived from the experimental and control group were detected by western blot analysis. The $\beta$-actin antibody was used as a loading control. Results are represented as the mean \pm standard deviation. ${ }^{*} \mathrm{P}<0.05$ indicates statistical significance.

size of the tumors (Fig. 6A, C and D), while the body weight of the tumor-bearing mice seemed not to be affected (Fig. 6B). In addition, we observed that there were no differences in the liver, kidney, intestine and brain of the tumor-bearing mice compared with the control group (data not shown). Thus, it was demonstrated that EA is a safe, nontoxic natural dietary product. Furthermore, we detected the influence of EA on xenograft cell proliferation by IHC and WB. As a result, we found that EA inhibited xenograft cell proliferation compared with the control group through the suppression of PCNA and Ki67 (Fig. 7).

EA regulates cell cycle-related proteins and members of the Bcl-2 family in xenograft tissues. Cell cycle-related and Bcl-2 member proteins largely determine the survival of cells (19). In this study, the influence of EA on the protein expression 


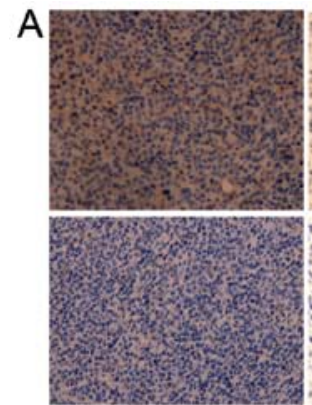

Bcl-2

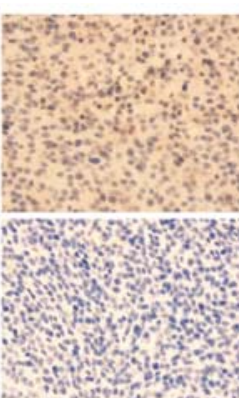

Cyclin D1
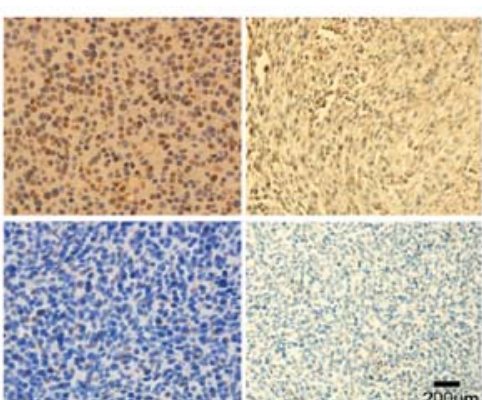

CDK2

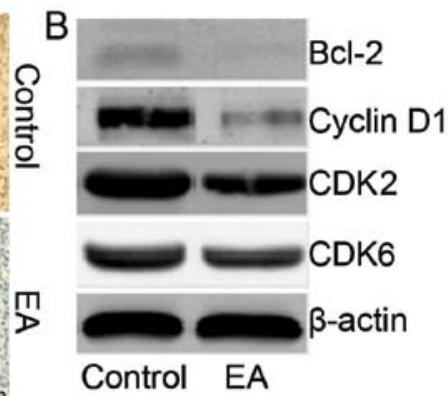

Control EA

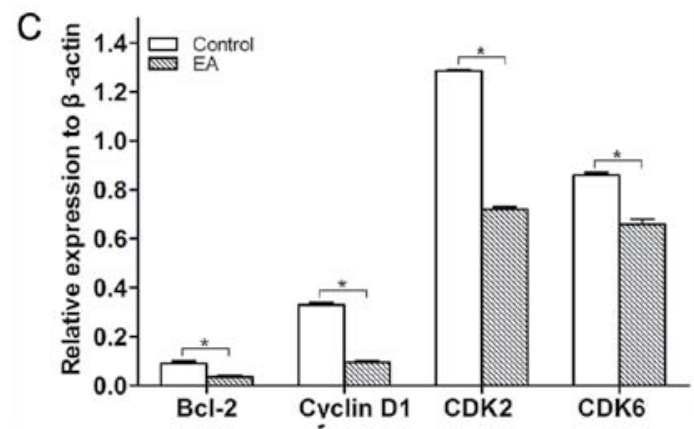

Figure 8. (A) Expression of B-cell lymphoma 2 (Bcl-2), cyclin D1, cyclin-dependent kinase (CDK)2, and CDK6 in xenografts derived from the experimental and control group was detected by immunohistochemistry. (B and C) The protein expression levels of Bcl-2, cyclin D1, CDK2, and CDK6 were detected by western blot analysis. The $\beta$-actin antibody was used as a loading control. Results are represented as the mean \pm standard deviation. ${ }^{*} \mathrm{P}<0.05$ indicates statistical significance.

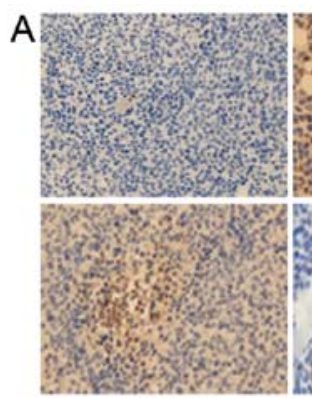

E-cadherin

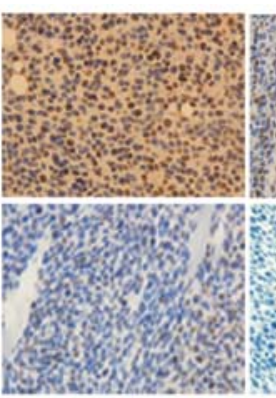

MMP-2
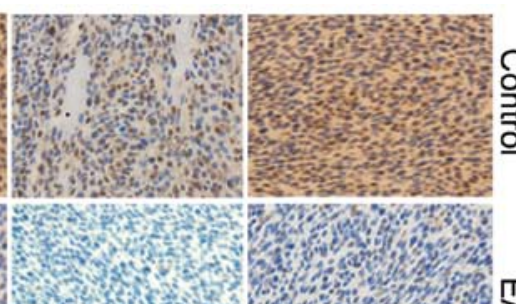

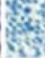

MMP-9

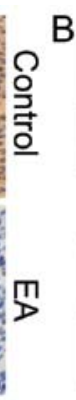

Snail

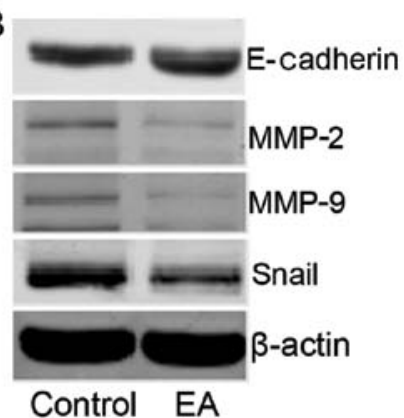

Control EA

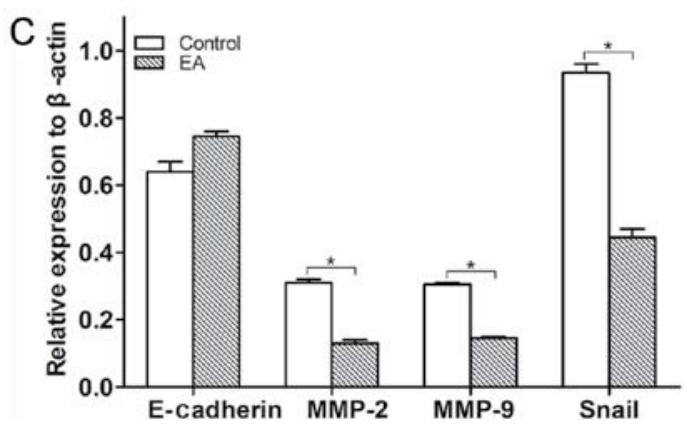

Figure 9. (A) The expression of E-cadherin, Snail, matrix metalloproteinase (MMP)-2 and MMP-9 in xenografts derived from the experimental and control group was detected by immunohistochemistry. (B and C) The protein expression level of E-cadherin, Snail, MMP-2 and MMP-9 in xenografts derived from the experimental and control group was detected by western blot analysis. The $\beta$-actin antibody was used as a loading control. Results were represented as mean \pm standard deviations. ${ }^{*} \mathrm{P}<0.05$ indicates statistical significance.

of cell cycle-related proteins and Bcl-2 family members from xenograft tissues was detected by IHC and WB. As a result, we found that EA suppressed the expression of Bcl-2 accompanied by the suppression of the protein expression of cyclin D1, CDK2 and CDK6 cell cycle-related proteins in the xenograft tissues (Fig. 8). Thus, it was revealed that EA regulates the cell cycle in U87 xenografted tumors.

EA suppresses epithelial-mesenchymal transition in xenograft tissues. During tumor cell invasion and metastasis, 
epithelial-mesenchymal transition (EMT) involved in embryonic development is usually activated (20). The process of EMT inhibits the expression of E-cadherin, while it induces and enhances the expression of transcription factors such as Snail and the expression of MMPs (21-24). In this study, the influence of EA on the protein expression of invasion was detected by IHC and WB. As a result, we found that EA upregulated the protein expression level of E-cadherin, while it suppressed the protein expression levels of Snail, MMP-2 and MMP-9 in xenograft tissues (Fig. 9). Thus, it was revealed that EA suppresses glioblastoma invasion by regulating the expression of EMT-associated transcription factors and MMPs.

EA inhibits the Akt and Notch pathways. The PI3K/Akt and Notch signaling pathways play an important role in cell survival, proliferation and progression. In this study, the influence of EA on the protein expression levels of Akt and Notch in xenograft tissues was determined by IHC and WB. As a result, we found that EA regulates U87 xenograft growth by inhibiting the Akt and Notch signaling pathways (Fig. 7).

\section{Discussion}

Glioblastoma is still a lethal human malignant tumor with limited progress in its treatment (25). Since EA was extracted from various plants hundreds of years ago, researchers have been increasingly attracted by its pharmacological properties (26). Several studies have shown that EA plays a significant role in the inhibition of cell proliferation and invasion in tumor cells. In the present study, we treated human glioblastoma U87 and U118 cells with EA. The results demonstrated that EA exhibited an inhibitory effect on cell viability, proliferation and invasion of human glioblastoma cells. Previous studies have illustrated that cell cycle arrest is associated with the inhibition of tumor cell proliferation. Many chemical reagents lead to cell DNA damage that inhibit cell proliferation. The DSBs induced by chemical reagents or ionizing radiation rapidly recruits a large amount of phosphorylated histone $\mathrm{H} 2 \mathrm{AX}$ named $\gamma-\mathrm{H} 2 \mathrm{AX}$, which exerts an essential role in DNA DSB repair (27). In a set of experiments, we observed cell cycle redistribution in both U87 MG and U118 MG cells. Simultaneously, the increased $\gamma-\mathrm{H} 2 \mathrm{AX}$ foci spots in the experimental group at $48 \mathrm{~h}$ showed that EA led to DNA damage and DSBs. Consequently, the cell cycle arrest and DNA damage induced by EA may contribute to those inhibitory results.

The Akt signaling pathway plays a major role in the suppression of cell apoptosis and facilitation of cell survival in malignant tumor cells (28). Akt signaling promotes cell survival by direct or indirect interaction with proteins linked with proliferation and apoptosis, such as anti-apoptotic factor Bcl-2 and cell cycle-associated protein cyclin D1. Furthermore, we found that EA inhibited glioblastoma U87 xenografted tumor growth which was involved in the inhibition of the Akt and Notch signaling pathways. The Akt and Notch signaling pathways play an important role in tumor carcinogenesis. Recently, the use of inhibitors of PI3K/Akt to treat cancer patients clinically is being assessed and more and more researchers are beginning to pay close attention to the therapeutic strategies in the suppression of PI3K/Akt activity. In this study, we found that EA suppressed tumor cell proliferation through the inhibition of the activation of Akt in human glioblastoma U87 xenografted tissues.

With activation of $\gamma$-secretase constitutively, aberrant Notch signaling plays a critical role in the initiation and progression of malignant tumors, and the inhibitors of this signaling pathway are presently being studied in clinical trials. A previous study showed that inhibition of Notch expression could decrease PI3K/Akt activity, which displays its antiproliferative effects from another point of view (29). Thus, suppression of the Notch signaling pathway alone or in combination with PI3K/Akt may be conducive to obtain maximum therapeutic effects. In this study, we found that EA inhibited tumor growth through the suppression of the Notch1 and Akt signaling pathways.

It is widely known that tumor cells undergoing EMT may exhibit the increase of specific secretion factors, such as cytokines, growth factors and chemokines, which could be beneficial for tumor progression (30). As a family of zinc-dependent proteases, MMPs are in a position to degrade the components of ECM, which promotes tumor invasion. In the present study, we observed that EA suppresses glioblastoma cell invasion in the Matrigel invasion assay, furthermore, EA inhibited glioblastoma invasion in the U87 xenografted model by inducing E-cadherin and suppressing the expression of transcription factors such as Snail, MMP-2 and MMP-9. All these findings reveal that EA suppresses metastasis in glioblastoma.

In conclusion, the suppression of the Akt and Notch pathways by EA may act together to inhibit glioblastoma growth, which suggests that EA, as a nontoxic compound, may possibly be employed for the treatment of glioblastoma in the future.

\section{Acknowledgements}

This study was supported by the National Natural Science Foundation of China (nos. 81372683 and 81572489 awarded to Q.C.). We acknowledge the assistance of the technical staff of the Key Laboratory of Renmin Hospital, Wuhan University, China.

\section{References}

1. Stupp R, Mason WP, van den Bent MJ, Weller M, Fisher B, Taphoorn MJ, Belanger K, Brandes AA, Marosi C, Bogdahn U, et al; European Organisation for Research and Treatment of Cancer Brain Tumor and Radiotherapy Groups; National Cancer Institute of Canada Clinical Trials Group: Radiotherapy plus concomitant and adjuvant temozolomide for glioblastoma. $\mathrm{N}$ Engl J Med 352: 987-996, 2005.

2. Cuddapah VA, Robel S, Watkins S and Sontheimer H: A neurocentric perspective on glioma invasion. Nat Rev Neurosci 15: 455-465, 2014

3. Yang JA, Li JQ, Shao LM, Yang Q, Liu BH, Wu TF, Wu P, Yi W and Chen QX: Puerarin inhibits proliferation and induces apoptosis in human glioblastoma cell lines. Int J Clin Exp Med 8: 10132-10142, 2015

4. Lee DH, Kim DW, Jung CH, Lee YJ and Park D: Gingerol sensitizes TRAIL-induced apoptotic cell death of glioblastoma cells. Toxicol Appl Pharmacol 279: 253-265, 2014.

5. Son YG, Kim EH, Kim JY, Kim SU, Kwon TK, Yoon AR, Yun $\mathrm{CO}$ and Choi KS: Silibinin sensitizes human glioma cells to TRAIL-mediated apoptosis via DR5 up-regulation and down-regulation of c-FLIP and survivin. Cancer Res 67: 8274-8284, 2007. 
6. Vattem DA and Shetty K: Biological function of ellagic acid: A Review. J Food Biochem 29: 234-266, 2005.

7. Zaveri NT: Green tea and its polyphenolic catechins: Medicinal uses in cancer and noncancer applications. Life Sci 78: 2073-2080, 2006.

8. Seeram NP, Adams LS, Henning SM, Niu Y, Zhang Y, Nair MG and Heber D: In vitro antiproliferative, apoptotic and antioxidant activities of punicalagin, ellagic acid and a total pomegranate tannin extract are enhanced in combination with other polyphenols as found in pomegranate juice. J Nutr Biochem 16 360-367, 2005

9. Han DH, Lee MJ and Kim JH: Antioxidant and apoptosis-inducing activities of ellagic acid. Anticancer Res 26 (5A) 3601-3606, 2006.

10. Strati A, Papoutsi Z, Lianidou E and Moutsatsou P: Effect of ellagic acid on the expression of human telomerase reverse transcriptase (hTERT) $\alpha+\beta+$ transcript in estrogen receptor-positive MCF-7 breast cancer cells. Clin Biochem 42: 1358-1362, 2009.

11. Saiko P, Steinmann MT, Schuster H, Graser G, Bressler S, Giessrigl B, Lackner A, Grusch M, Krupitza G, Bago-Horvath Z, et al: Epigallocatechin gallate, ellagic acid, and rosmarinic acid perturb dNTP pools and inhibit de novo DNA synthesis and proliferation of human HL-60 promyelocytic leukemia cells: Synergism with arabinofuranosylcytosine. Phytomedicine 22: 213-222, 2015.

12. Arulmozhi V, Pandian K and Mirunalini S: Ellagic acid encapsulated chitosan nanoparticles for drug delivery system in human oral cancer cell line (KB). Colloids Surf B Biointerfaces 110 313-320, 2013

13. Zhao M, Tang SN, Marsh JL, Shankar S and Srivastava RK Ellagic acid inhibits human pancreatic cancer growth in Balb c nude mice. Cancer Lett 337: 210-217, 2013.

14. Okla M, Kang I, Kim DM, Gourineni V, Shay N, Gu L and Chung S: Ellagic acid modulates lipid accumulation in primary human adipocytes and human hepatoma Huh7 cells via discrete mechanisms. J Nutr Biochem 26: 82-90, 2015.

15. Umesalma S and Sudhandiran G: Ellagic acid prevents rat colon carcinogenesis induced by 1,2 dimethyl hydrazine through inhibition of AKT-phosphoinositide-3 kinase pathway. Eur J Pharmacol 660: 249-258, 2011

16. Guo W, Li A, Jia Z, Yuan Y, Dai H and Li H: Transferrin modified PEG-PLA-resveratrol conjugates: In vitro and in vivo studies for glioma. Eur J Pharmacol 718: 41-47, 2013.
17. Purow B: Notch inhibition as a promising new approach to cancer therapy. Adv Exp Med Biol 727: 305-319, 2012.

18. Koch U and Radtke F: Notch and cancer: A double-edged sword. Cell Mol Life Sci 64: 2746-2762, 2007.

19. Cory S, Huang DC and Adams JM: The Bcl-2 family: Roles in cell survival and oncogenesis. Oncogene 22: 8590-8607, 2003.

20. Steeg PS: Tumor metastasis: Mechanistic insights and clinical challenges. Nat Med 12: 895-904, 2006.

21. Kraljevic Pavelic S, Sedic M, Bosnjak H, Spaventi S and Pavelic K: Metastasis: New perspectives on an old problem. Mol Cancer 10: 22, 2011

22. McCarthy N: Metastasis: Route master. Nat Rev Cancer 9: 610-611, 2009

23. McCarthy N: Metastasis: Influencing bad behaviour. Nat Rev Cancer 9: 609, 2009

24. Nguyen DX, Bos PD and Massagué J: Metastasis: From dissemination to organ-specific colonization. Nat Rev Cancer 9: 274-284, 2009.

25. Grossman SA and Batara JF: Current management of glioblastoma multiforme. Semin Oncol 31: 635-644, 2004

26. Landete JM: Ellagitannins, ellagic acid and their derived metabolites: A review about source, metabolism, functions and health. Food Res Int 44: 1150-1160, 2011.

27. Hernández L, Terradas M, Martín M, Tusell L and Genescà $A$ : Highly sensitive automated method for DNA damage assessment: gamma-H2AX foci counting and cell cycle sorting. Int J Mol Sci 14: 15810-15826, 2013.

28. Koseoglu S, Lu Z, Kumar C, Kirschmeier P and Zou J: AKT1, AKT2 and AKT3-dependent cell survival is cell line-specific and knockdown of all three isoforms selectively induces apoptosis in 20 human tumor cell lines. Cancer Biol Ther 6: 755-762, 2007.

29. Wang D, Chen Q, Liu B, Li Y, Tan Y and Yang B: Ellagic acid inhibits proliferation and induces apoptosis in human glioblastoma cells. Acta Cir Bras 31: 143-149, 2016.

30. Palena C, Hamilton DH and Fernando RI: Influence of IL-8 on the epithelial-mesenchymal transition and the tumor microenvironment. Future Oncol 8: 713-722, 2012. 